\title{
Unavoidable caesarean myomectomy A case report
}

\author{
*1Alexander T Owolabi, 'Olabisi M Loto, 'Oluwafemi Kuti, \\ ${ }^{2}$ Richard $\mathbf{R}$ Ehinmitan \& ${ }^{2}$ Ayuba $\mathbf{Y}$ Ibrahim \\ 'Department of Obstetrics, Gynecology and Perinatology, Obafemi Awolowo University \& \\ ${ }^{2}$ Department of Obstetrics, Gynecology, Obafemi Awolowo University Teaching Hospitals \\ Complex Ile-Ife, Osun State
}

\begin{abstract}
Establishment of the feasibility and need to perform myomectomy during caesarean section when fibroid obstruct wound closure with associated hemorrhage has been presented.

A case of a primigravida with a huge uterine fibroid located in the lower segment lifting up the uterine cavity leading to persistent transverse lie of the fetus at term is presented. An elective classical caesarean section was performed with difficulty in uterine wound closure and haemorrhage necessitating myomectomy. Haemorrhage was controlled with the use of Foley catheter tourniquet and high dose oxytocin infusion. Literature review was carried out. The mother and baby had satisfactory outcome.

To conclude, life threatening situations of myomectomy with caesarean section ought to be, and can be safely performed using tourniquet and high dose oxytocin to reduce haemorrhage. The uterus in the immediate post partum period is better adapted physiologically to control haemorrhage than any other stage of a woman's life.
\end{abstract}

Key Words: Classical caesarean Section, Myomectomy, caesarean-myomectomy, Haemorrhage.

\section{Introduction}

Uterine lieomyomata or fibroids are the commonest tumours of the female genital tract. It is 3-9 times commoner among the Negroid race, compared to the Caucasians ${ }^{1}$. Traditionally obstetricians and gynaecologist had avoided performing myomectomy either during pregnancy or at cesarean section because of fear that bleeding may be intractable as a result of the increased vascularity of the pregnant uterus. The presence of fibroid in the lower uterine segment may be an indication for classical caesarean section. Recent reports indicate that myomectomy, at the time of caesarean section operation; can be safely undertaken by skilled practitioners and with the application of tourniquet at the base of the broad ligament or infusion of high dose oxytocin to control haemorrhage ${ }^{2-5}$. A case is presented of an unavoidable myomectomy performed during caesarean section for persistent transverse lie at term due to a huge uterine fibroid filling the whole of the lower uterine segment.

\section{Case}

Mrs M.O. is a 35 years old prigravida who booked for antenatal care at the Wesley Guild Hospital Unit of Obafemi Awolowo University Teaching Hospitals Complex (OAUTHC), Ilesa at gestational age of 17 weeks. The uterine fundal height at booking was larger than the gestational age by date. An ultrasound scan performed revealed a singleton foetus in longitudinal lie with breech presentation with adequate amniotic fluid. There was a fibroid mass measuring 16 x $12 \times 8 \mathrm{~cm}$ anteriorly placed in the lower uterine segment (Fig 1). The antenatal period remained uneventful until 35 weeks gestation when the fetal lie was found to be transverse. The transverse lie persisted at 37 weeks and she was offered elective caesarean section at 38

\section{Correspondence}

Dr. A.T. Owolabi,MBBS, M.Comm.H, FWACS

Department of Obstetrics, Gynecology and Perinatology, College of Health Sciences, Obafemi Awolowo University, Ile Ife, Osun State, Nigeria, Tel: +234 803715 1125. FAX 234-36-230-705,

Email: alexandrerowolabi@yahoo.com 


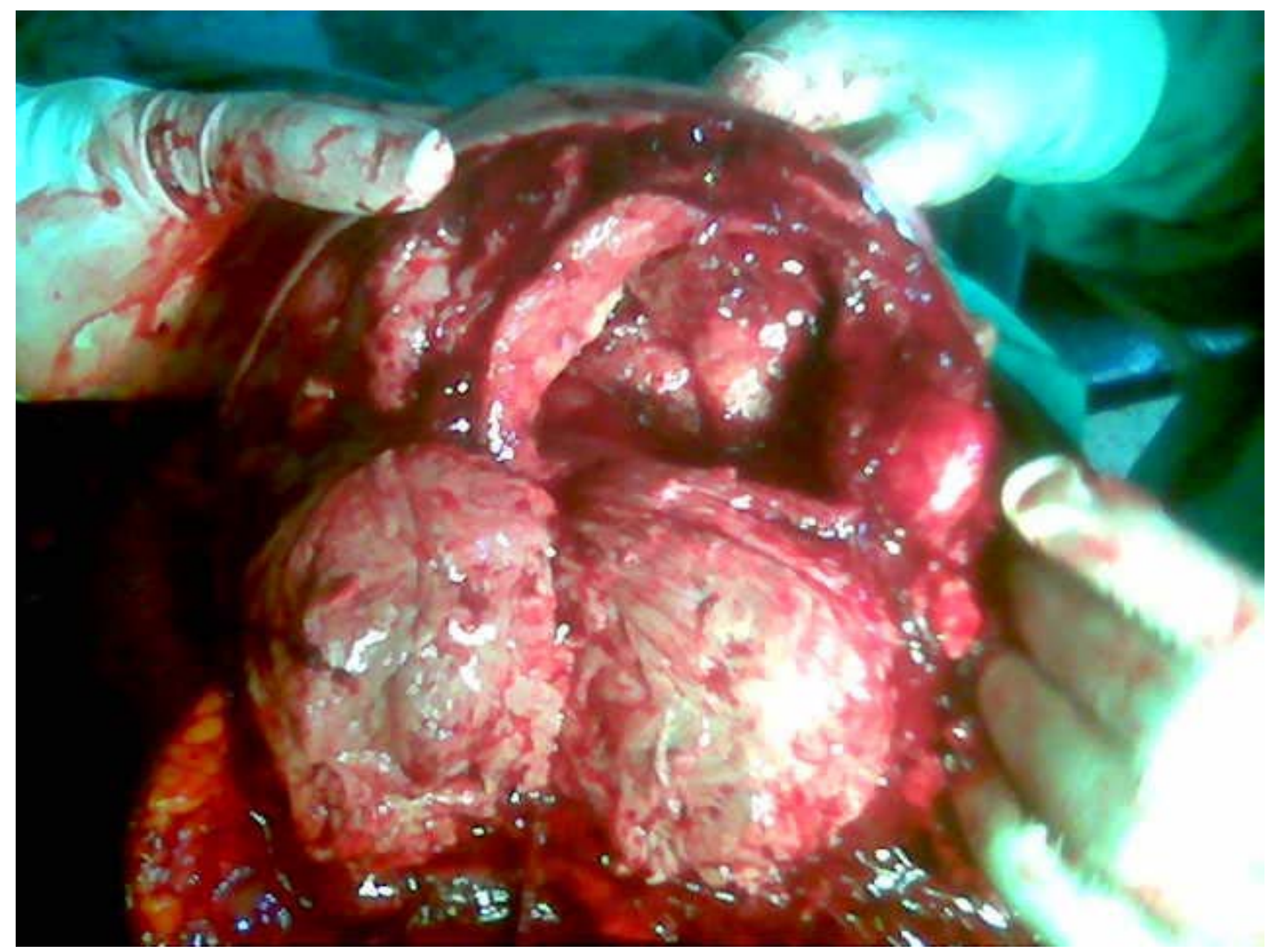

Figure I. Uterus with a big intramural fibroid. The photograph is JPEG format. It shows a big fibroid occupying the lower uterine segment completely lifting up the uterine cavity

weeks. Pre-operative haemoglobin concentration was $11.5 \mathrm{~g} / \mathrm{dl}$. Two units of blood screened for HIV and Hepatitis B \& C, were grouped and crosshatched for her use.

The fibroid occupied the lower uterine segment completely lifting up the uterine cavity above it with the baby in transverse lie necessitating the use of classical caesarean section to deliver the baby (Fig 1).

Alife female baby weighing $3.5 \mathrm{Kg}$ and Apgar score 8 at one minute and 10 at ten minutes was delivered. The anteriorly located intramural uterine fibroid mass measuring $16 \times 13 \times 9 \mathrm{~cm}$ made wound closure impossible with associated profuse bleeding from the wound site (Figure 1). High dose intravenous oxytocin infusion (60 i.u. in $500 \mathrm{mls}$ of $5 \%$ dextrose in water) was set up to ensure adequate uterine contraction. A tourniquet was applied to the lower part of the broad ligament to compress both uterine arteries and the vessels in the infundibulo-pelvic ligament so as to achieve a bloodless operating field. The fallopian tubes were lifted and excluded from the tourniquet. The tourniquet insertion time was noted. The fibroid was dissected and enucleated and the cavity was closed in three layers. The classical uterine incision closure was then completed effectively. The estimated blood loss was 1.3 liters. Oxytocin infusion was continued for 24 hours post postoperatively. Prophylactic antibiotics and analgesics were given.

Post operatively the patient remain stable however on the $3^{\text {rd }}$ postoperative day her haemoglobin concentration was $8 \mathrm{~g} / \mathrm{dl}$ while the packed cell volume was $24 \%$. She was transfused with two pints of blood to correct the anemia. Subsequent post-operative period remained uneventful she was discharged home on the $8^{\text {th }}$ post-operative day with hemoglobin concentration of $10.5 \mathrm{~g} / \mathrm{dl}$ and a packed cell volume of $30 \%$.

\section{Comment}

Management of fibroids during caesarean delivery remained controversial. Although the standard practice is to avoid myomectomy during caesarean delivery, under certain conditions cesarean myomectomy becomes unavoidable ${ }^{3,4,6}$. This case illustrates the need for caesarean myomectomy because of the threat to the life of the patient from haemorrhage arising from the difficulty with wound closure due to the huge lower uterine intramural fibroid. The alternative of caesarean 
hysterectomy would have been catastrophic for a young woman having her first baby. Blood loss in this patient was as a result of the recourse to classical caesarean section due to transverse lie of the foetus and the difficulty in wound closure. The uterine cavity was above the huge uterine fibroid and made it inaccessible even if the fibroid was enucleated before the delivery of the baby. This is one of the indications for classical caesarean section ${ }^{7}$. Prompt application of Foleys catheter tourniquet tied round to encompass and compress both uterine arteries at the base of the broad ligament and the vessels in the infundibulo-pelvic ligament adequate control of bleeding for the rest of the surgery was ensured. Other measures to control haemorrhage included the use of high dose oxytocin infusion and the meticulous closure of the cavity created by the removal of the fibroid in three layers. A study carried out in Ghana by Kwawukume ${ }^{2}$, (2002) confirmed the efficacy and safety of myomectomy done at caesarean section with the application of a tourniquet compared to cesarean section without myomectomy with the conclusion that there was no significant difference in intra-operative and postoperative morbidity, and blood loss between the two groups.

Though routine myomectomy during caesarean section is not a standard practice it ought to be, and can be safely performed in selected cases in experienced hands with the advantage of avoiding the need for interval myomectomy especially when more pregnancies are desired. This will help to eliminate other associated problems of fibroid such as anaemia, dysmenorrhoea, degenerative changes and complications during subsequent pregnancies.
The uterus in the immediate post partum period is better adapted physiologically to control haemorrhage than any other stage of a woman's life.

\section{References}

1. Ogedengbe OK. Uterine fibroids. In: Okonofua F, Odunsi K (eds.), Contemporary Obstetrics and Gynaecology for developing Countries, Benin City: Women's Health and Action Research Centre. 2003; 202-213.

2. Kwawukumen EY. Caesarean Myomectomy. Afr J Reprod Health. 2002; 6(3): 38-43.

3. Ehigiegba AE, Anda, AB, Ojobo SI. Myomectomy during Caesarean Section. Int. J. Gynecol Obstet. 2001; 75:21-25.

4. Agboghoroma CO, Efetie ER and Umezurlike AC Unavoidable Caesarean Myomectomy: A Case Report. Trop J Obstet Gynaecol. 2005; 22(1): 8182.

5. Sudhir A. and Sebanti G. Cesarean myomectomyA study of 14 cases. The Journal of Obstetrics and Gynaecology of India. 2006; 56 (6):486-488.

6. Ande A.B. Ehigiegba A.E. and Umeora O.U. Repeat myomectomy at caesarean section. Arch Gynaecol Obstet. 2004; 270 (4):296-8.

7. Bolaji I.I., Rafla N.M. and Mylotte M.J. Classical caesarean section through the posterior uterine wall. Ir J Med Sci. 1992; 161 (2):46-7 